\title{
El sistema regional de innovación y la política tecnológica: el caso de Tamaulipas, México
}

\section{Technology Policy and the Regional Innovation System: A Case Study in Tamaulipas, México}

\author{
José Luis López Gómez* (D) https://orcid.org/0000-0002-2279-0440 \\ Francisco García Fernández ${ }^{* *}$ (1) https://orcid.org/0000-0003-4340-1093
}

\section{Resumen}

Objetivo: caracterizar el sistema regional de innovación y la política tecnológica regional implementada en Tamaulipas desde 2016 hasta 2020. Metodología: investigación descriptiva de los indicadores cuantitativos a través de representaciones gráficas longitudinales y transversales, con el propósito de detectar las principales deficiencias del sistema mediante la clasificación de fallas que proponen Tödtling y Trippl de los sistemas regionales de innovación. Resultados: las principales fallas son la ausencia de vinculación entre los agentes involucrados y la carencia de objetivos claros de las instituciones rectoras. Limitaciones: la falta de consenso generalizado para definir un conjunto de indicadores únicos para estudiar los sistemas regionales de innovación en México, lo que queda pendiente para futuros estudios. Conclusiones: a pesar de los avances y del posicionamiento de los principales indicadores, la política tecnológica regional no ha tenido una implementación rigurosa, un seguimiento adecuado o una visión a largo plazo. Por lo tanto, no hay una estrategia de desarrollo económico estatal.

Palabras clave: sistema regional de innovación; política tecnológica; Tamaulipas.

\begin{abstract}
Objective: to characterize the regional innovation system and the regional technology policy implemented in Tamaulipas from 2016 to 2020. Methodology: description of the quantitative indicators throughout longitudinal and cross-sectional graphical representations to detect the principal deficiencies of the system by using the classification of failures proposed by Tödtling and Trippl. Results: the central deficiencies are the lack of linkage between the agents involved and the absence of clear objectives among the governing institutions. Limitations: the lack of generalized consensus to define a set of unique indicators to study the regional innovation systems in Mexico, which is pending for future studies. Conclusions: despite the advances and positioning of the main indicators, the regional technology policy has not had a rigorous implementation, an adequate follow-up, or a long-term vision. Hence, there has not been an state's economic development strategy.

Keywords: regional innovation system; technology policy; Tamaulipas.
\end{abstract}

Cómo citar: López Gómez, J. L., y García Fernández, F. (202I). El sistema regional de innovación y la política tecnológica: el caso de Tamaulipas, México. región y sociedad, 33, el4 I0. doi: 10.22198/rys2021/33/I4I0

*Autor para correspondencia. Investigador independiente. Ciudad Victoria, Tamaulipas, México. Correo electrónico: lopez-josel@hotmail.com

** Universidad Autónoma de Tamaulipas, Centro Universitario Victoria. Bulevar Adolfo López Mateos s. n., Col. Centro, C. P. 87149, Ciudad Victoria, Tamaulipas, México. Correo electrónico: ffernandez@docentes.uat.edu.mx

Recibido: 11 de octubre de 2020

Reevaluado: 16 de marzo de 2021

Aceptado: 18 de mayo de 2021

Liberado: 10 de junio de 2021

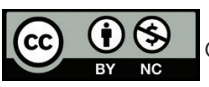

Esta obra está protegida bajo una Licencia Creative Commons Atribución-No Comercial 4.0 Internacional. 


\section{Introducción}

Hay un factor común entre los países que han obtenido los mayores niveles de desarrollo económico y bienestar social. Esas naciones han invertido sus recursos financieros y humanos en ciencia, tecnología e innovación, y han desarrollado modelos que promueven la producción de nuevos conocimientos y su aplicación a todos los ámbitos de la vida. Se ha dado un consenso en el mundo académico y político respecto a la importancia de la innovación y del aprendizaje como fuentes de competitividad, puesto que se considera que la habilidad para innovar es la base del desarrollo de un territorio (Comisión Económica para América Latina y el Caribe [CEPAL], 2000).

En los últimos dos decenios ha habido un esfuerzo particular en el mundo académico para estudiar los procesos de innovación a través de una perspectiva regional (Carlsson, 2007; Morgan, 1997; Niembro, 2019). Esa línea de investigación ha llevado a la proliferación de trabajos que estudian las particularidades de los sistemas de innovación regional en distintos contextos (Doloreux y Porto, 2017).

Los gobiernos desempeñan un papel fundamental en la implementación y en la ejecución de las acciones públicas que se orientan al desarrollo y al fortalecimiento de los procesos de innovación (Heijs, 2001). Esas medidas de intervención han tenido en México resultados limitados y desiguales a escala regional. A partir de la década de 1980, con el tránsito al modelo neoliberal y el repliegue del Estado de la mayoría de las actividades económicas, el mercado y los agentes privados ocupan una posición determinante en la definición de las estrategias para el desarrollo tecnológico (Cimoli, Ferraz y Primi, 2007). La acción pública se limita a corregir las fallas de mercado según las prescripciones de la teoría económica convencional y a construir instituciones para un modelo en el que el mercado es el principal regulador y el Estado se posiciona como institución subsidiaria.

Tamaulipas es uno de los estados fronterizos de significativa relevancia para la economía de México. Aporta $2.74 \%$ del producto interno bruto (PIB) nacional (2018), lo cual lo ubica en la posición decimosegunda a escala nacional (Instituto Nacional de Estadística y Geografía [INEGI], 2019a). Esta contribución llegó a su máximo en 2005 (3.10\% del PIB), pero su porcentaje disminuyó a partir de esta fecha debido en parte al estancamiento continuo de la economía estatal. Entre 2000 y 2019, el PIB estatal per cápita experimentó una tasa media anual de crecimiento de $0.58 \%$ (INEGI, 2019a). Alrededor de 2010 se acentuó la violencia derivada de las actividades del crimen organizado, lo que favoreció la propagación de un clima negativo de inversión, adverso a cualquier tipo de actividad empresarial en las ciudades y zonas rurales del estado. Una diversidad de factores ha influido para este desempeño de la economía tamaulipeca desde hace dos décadas. Como han señalado Lundvall, Vang, Joseph y Chaminade (2009), la mejora de las economías depende de los sistemas de innovación, pues su objetivo último no es elevar la producción de innovaciones per se, sino maximizar el bienestar económico. Precisamente, a diferencia de otras entidades (Fuentes y Ampudia, 2009; López, Moreno, Marín y Maldonado, 2013; 
Ordóñez, 2017; Rosales y López, 2008), Tamaulipas ha carecido de una política tecnológica regional orientada a fortalecer los atributos propios de su sistema de innovación, lo que se constata en la falta de una estrategia de desarrollo regional y en un enfoque de sistemas de innovación en sus instituciones.

Este trabajo tiene como objetivo analizar las condiciones del sistema regional de innovación (SRI) y de la política tecnológica regional implementada en Tamaulipas en los últimos años, mediante el estudio de la actuación de los principales agentes involucrados, de sus funciones y de los resultados obtenidos. El artículo se divide en cuatro secciones. En la primera se presenta una revisión teórica del SRI y el papel de la política tecnológica regional en éste. La segunda parte expone la metodología utilizada. En la tercera se abordan los resultados del desempeño de los actores del SRI y las principales fallas que limitan su funcionamiento. En la última sección se ofrecen las conclusiones, en las que se responden las siguientes interrogantes: ¿Cuáles son los agentes que componen el SRI en Tamaulipas?, ¿cuáles son sus principales problemas? y ¿existe una política tecnológica regional en Tamaulipas?

\section{Sistema de innovación regional y política tecnológica}

El enfoque del sistema de innovación surge a finales de la década de 1970 como resultado de los esfuerzos de un grupo de investigadores que buscaban explicar los procesos de globalización y cambio tecnológico desde una perspectiva heterodoxa. Dichos esfuerzos se debieron a su vez a la incapacidad de la economía convencional para comprender esos procesos de manera satisfactoria, ya que eran considerados tan sólo variables exógenas dentro de los modelos clásicos de crecimiento económico (Solow, 1956).

Para la economía neoclásica tradicional, en determinadas circunstancias, no hay incentivos suficientes en el mercado para que tenga lugar el desarrollo tecnológico. Desde esta perspectiva, la incertidumbre, las externalidades y las ventajas de escala son distorsiones del mercado que atentan contra el funcionamiento eficiente del proceso de innovación (Arrow, 1962). El mismo Arrow (1962) enfatizó la discrepancia entre las conclusiones del modelo y el mundo real, además de contribuir a la comprensión del rol del cambio tecnológico en la economía.

El sistema de innovación fue un concepto de origen neoschumpeteriano y pretendió expresar la complejidad misma de este fenómeno sugiriendo que la innovación no es producto de esfuerzos aislados, sino el resultado de las interacciones entre los agentes participantes en un contexto institucional determinado (Quintero, 2010). El trabajo de Freeman (1987) sobre Japón sentó las bases para investigar la política tecnológica y el sistema de innovación. En dicho estudio, los sistemas de innovación ponen énfasis en la intensidad de las relaciones entre los participantes y en las relaciones con las instituciones públicas y privadas encargadas de difundir el conocimiento.

Lundvall (1992) describe los sistemas de innovación como el conjunto de factores que interactúan en la generación, uso y divulgación de nuevos cono- 
cimientos con aprovechamiento económico. Los sistemas de innovación no son estáticos; evolucionan con la configuración productiva, las instituciones y el nivel de desarrollo (Cooper, 1999; Gu, 1999). Por consiguiente, sería complicado hallar un modelo perfecto que encaje en varios países o sociedades.

A partir de la literatura de los sistemas de innovación (SI), el concepto de sistema nacional de innovación (SNI) toma mayor fuerza debido a las obras de Nelson (1992), de Dosi, Freeman, Nelson, Silverberg y Soete (1988) y de Lundvall, Johnson, Andersen y Dalum (2002). El desarrollo conceptual significó un avance importante al incorporar la estructura institucional y el esfuerzo para estimular la actividad de investigación y desarrollo (I+D) como el sostén del desarrollo competitivo a escala nacional (Lundvall, Johnson, Andersen y Dalum, 2002). Entre las principales definiciones de los SNI, se encuentra la que propone Freeman (1987), quien los explica como el entramado de instituciones privadas y públicas en las que sus acciones e interacciones comienzan, introducen, alteran y promueven nuevas tecnologías. Más adelante, Patel y Pavitt (2000) incluirían las competencias, los estímulos y las fallas de mercado en el concepto, al considerar que el valor del aprendizaje tecnológico de un país está determinado por las estructuras de estímulos y el nivel de competencia de las instituciones nacionales.

En ese entorno surge el término sistema regional de innovación, un concepto derivado del SNI, con la pretensión de reflejar las heterogeneidades regionales que éste no capta. Por eso el término sería definido como el conjunto de actores, vínculos e instituciones que interactúan en relación con la producción y la difusión de conocimientos en un territorio (Cooke, 2001; Doloreux, 2002; Quintero, 2010). El concepto de SRI expresa la consolidación de los análisis que buscan entender los determinantes de la innovación a escala regional, los cuales son muy diferentes a los procesos de innovación que se producen a escala nacional. De manera similar, el surgimiento del SRI está asociado con los trabajos de Cooke (1992), quien lo define como un sistema "en el que las empresas y otras organizaciones participan sistemáticamente en el aprendizaje interactivo a través de un entorno institucional caracterizado por la integración" (Cooke, Uranga y Etxebarria, 1998, p. 22). Este enfoque introduce el aprendizaje institucional y la comprensión de la interacción entre los agentes participantes del proceso innovador.

Asheim e Isaksen (1997) enfatizarían más tarde que un SRI está conformado por estructuras interrelacionadas, las que luego se redefinieron como subsistemas: la estructura de producción (estructuras tecno-económicas) y la infraestructura institucional (estructuras político-institucionales). La conceptualización del SRI como conjunto de subsistemas es tal vez la definición más aceptada en la actualidad. Según esta definición, el SRI está formado por un conjunto de subsistemas. Los dos subsistemas claves son: el subsistema de creación y producción de conocimientos y el subsistema de explotación del conocimiento (Cooke, Roper y Wylie, 2003).

A pesar de las particularidades de cada economía, existe el consenso de que un SRI exitoso debe contener los siguientes componentes (Autio, 1998; Komninaki, 2015): 
- Subsistema de generación y difusión del conocimiento: compuesto por universidades, centros de investigación, laboratorios de investigación y centros de formación, entre otros.

- Subsistema de explotación y aplicación del conocimiento: estructura de producción regional compuesta por empresas.

- Subsistema de instituciones gubernamentales y agencias especializadas para el desarrollo regional (Tödtling y Trippl, 2007).

- Interacciones intensivas entre subsistemas, en términos de conocimiento científico y flujos de recursos humanos, incluidos vínculos con otras instituciones regionales y nacionales.

Con el propósito de reconocer cuáles son las principales fallas del SRI de un territorio, Tödtling y Trippl (2005) elaboraron una clasificación de tres elementos. El primer componente se refiere a la debilidad institucional; en otras palabras, a la escasez de organismos e instituciones que lleven a cabo acciones de política pública de alto impacto para el desarrollo regional. El segundo es la ausencia de vínculos entre los subsistemas, carencia que se caracteriza no sólo por considerar las pocas interrelaciones entre los agentes de los sectores académicos y productivos, sino también por contemplar la intensidad de éstas. El tercer componente se refiere al bloqueo, problemática resultante de una especialización en tecnologías maduras y una muy limitada producción de tecnologías emergentes.

En México, varios estudios han aplicado el concepto de SRI. Dutrénit (2009) abordó las características de cada uno de los sectores del SRI analizados desde una perspectiva local al resaltar la importancia de las pequeñas y medianas empresas (PYMES) en el sistema. Fuentes y Ampudia (2009) describieron las propiedades de los agentes que intervienen en las actividades de manufactura y su vínculo con las PYMES de las ciudades de Querétaro y Juárez. De la misma manera, Feria, Rodríguez y Herrera (2012) caracterizaron los actores institucionales y empresariales del sistema de Aguascalientes como un recurso para detectar la existencia de vínculos entre ellos. López et al. (2013) examinaron las contribuciones teóricas de diferentes autores al concepto y destacan el papel de la innovación como una estrategia de competitividad para el estado de Baja California. Rosales y López (2008) evaluaron el caso del SRI en Sinaloa y su relación con la capacidad exportadora del estado. Por último, Ordóñez (2017) estudia el caso del SRI de Jalisco y desde un enfoque de economía política critica la estrategia de desarrollo regional implementada.

En una parte de la literatura especializada reciente se utilizan con frecuencia, casi como sinónimos, política de innovación y política tecnológica. El enfoque tradicional concibe la política tecnológica como las acciones concentradas en fortalecer las áreas científico-técnicas, con el objetivo de promover las actividades de investigación y desarrollar las nuevas tecnologías industriales (Meyer-Krahmer, 1997). En Heijs (2001) dichas políticas son puestas en marcha por los gobiernos para promover transformaciones estructurales en la economía a través del cambio tecnológico y la creación de ventajas comparativas. 
Por el contrario, el más reciente enfoque concibe las políticas de innovación desde un punto de vista más amplio, que significa no delimitar dichas estrategias a las áreas de ciencia y tecnología, sino incluir además otros aspectos que comprendan los temas organizacionales, educativos, financieros, sectoriales, de infraestructura y de redes (Niembro, 2019; Tödtling y Trippl, 2005; 2011).

El objetivo básico de una política orientada al desarrollo tecnológico es contribuir a formar y mejorar los sistemas de innovación, ya sean nacionales, regionales o sectoriales, para consolidar un flujo continuo de innovaciones para el sector productivo y desplazar la frontera de conocimiento de la región (Heijs, 2001).

La teoría neoschumpeteriana considera que los esfuerzos de la política tecnológica deben apoyarse en el desarrollo deliberado de capacidades científicas y tecnológicas, dado que, en muchas ocasiones, las transferencias de conocimientos no son perfectas. Esta situación hace necesario el accionar público para fomentar la producción de conocimientos que no se demandan desde el ámbito privado por un déficit de incentivos. De tal modo que un aumento en la generación de conocimientos repercutirá en un incremento de la eficiencia y la productividad, con efectos en el establecimiento de una corriente continua de actividades innovadoras. Los agentes implicados en dichos procesos para lograr un beneficio social y económico han sido captados mediante el concepto de sistema de innovación (Chudnovsky, 1999). Por lo tanto, el desarrollo de un

Tabla 1. Acciones de la política tecnológica regional

\begin{tabular}{|c|c|c|c|}
\hline \multirow[b]{2}{*}{ 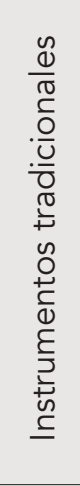 } & $\begin{array}{l}\text { Generación de } \\
\text { conocimiento }\end{array}$ & $\begin{array}{c}\text { Difusión de } \\
\text { conocimiento }\end{array}$ & $\begin{array}{l}\text { Explotación de } \\
\text { conocimiento }\end{array}$ \\
\hline & $\begin{array}{l}\text { - Fondos tecnológicos, } \\
\text { incentivos, apoyos o } \\
\text { subvenciones a I+D. } \\
\text { - Apoyo al desarrollo } \\
\text { de infraestructuras. } \\
\text { - Capital humano para } \\
\text { ciencia y tecnología } \\
\text { (CYT). }\end{array}$ & $\begin{array}{l}\text { - Parques científicos. } \\
\text { - Oficinas y programas } \\
\text { de transferencia } \\
\text { tecnológica. } \\
\text { - Planes de movilidad y } \\
\text { de atracción de talento. } \\
\text { - Agentes de innovación. } \\
\text { - Premios a la innovación. }\end{array}$ & $\begin{array}{l}\text { - Incubadoras de } \\
\text { empresas. } \\
\text { - Servicios innovadores a } \\
\text { empresas } \\
\text { (apoyo y coaching). } \\
\text { - Apoyo a la creación } \\
\text { de empresas. } \\
\text { - Formación y } \\
\text { sensibilización para la } \\
\text { innovación. }\end{array}$ \\
\hline 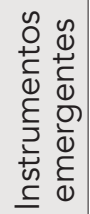 & $\begin{array}{l}\text { - Alianzas } \\
\text { público-privadas } \\
\text { para la innovación. } \\
\text { - Redes-polos de } \\
\text { investigación. }\end{array}$ & $\begin{array}{l}\text { - Cheques a la } \\
\text { innovación. } \\
\text { - Certificaciones o } \\
\text { acreditaciones. }\end{array}$ & $\begin{array}{l}\text { - Doctorados en industria } \\
\text { - Apoyo al diseño y a la } \\
\text { creatividad. }\end{array}$ \\
\hline \multirow{2}{*}{ 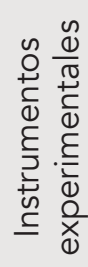 } & \multicolumn{3}{|c|}{$\begin{array}{l}\text { Polos de competitividad. } \\
\text { Fondos de capital inicial y capital de riesgo. } \\
\text { Sistemas de garantía para financiar la innovación. }\end{array}$} \\
\hline & $\begin{array}{l}\text { - Centros } \\
\text { transfronterizos } \\
\text { de investigación. }\end{array}$ & & $\begin{array}{l}\text { - Adquisición pública } \\
\text { regional de bienes } \\
\text { y servicios. }\end{array}$ \\
\hline
\end{tabular}

Fuente: elaboración propia con datos de la OCDE (2011). 
SRI exitoso no es posible sin una política de innovación de alcance regional. Esto significa que el diseño y la instrumentación de las acciones públicas deben potenciar los procesos de innovación en la dimensión regional (Llisterri, Pietrobelli y Larsson, 2011).

La Organización para la Cooperación y el Desarrollo Económicos (OCDE, 2011) ha sido clave en la creación de propuestas de política de innovación regional. La mayor experiencia en la implementación de acciones de esta índole se ha alcanzado dentro de la Unión Europea (Mas Verdú, 2014). En cambio, en México no hay trabajos previos que describan acciones de política tecnológica regional o experiencias en este campo. Dichas propuestas de la OCDE pretenden concentrarse en las relaciones de los agentes del sistema de innovación mediante la creación y la identificación de instituciones que promuevan la generación, la difusión y la explotación del nuevo conocimiento.

Para la consecución de estos objetivos, la OCDE (2011) proporciona una clasificación de instrumentos que buscan el desarrollo de las actividades de innovación a partir del estudio de dos dimensiones (véase tabla 1). Una de ellas se centra en el grado de aceptación y extensión de las acciones de tipo tradicional, emergentes y experimentales. La segunda refleja los objetivos a conseguir, tales como la generación, la difusión y la explotación de conocimiento.

\section{Metodología}

En la actualidad, tanto académicos como gobiernos reconocen la importancia de la innovación como fuente de competitividad para cualquier país y región. El objetivo de este trabajo es exponer las características de los agentes (industria, dependencias gubernamentales e instituciones académicas) que conforman el SRI en Tamaulipas y distinguir las interacciones entre ellos, con el fin de realizar una primera caracterización del sistema e identificar sus fortalezas y deficiencias predominantes. Para cumplir este objetivo se realizó una investigación descriptiva con diseño no experimental.

A pesar de que no existe un consenso generalizado para definir un conjunto de indicadores únicos que permitan estudiar los SRI (Doloreux y Parto, 2005; Tödtling y Trippl, 2005), se optó por aquellos que posibilitaran ilustrar de forma adecuada los atributos de la entidad. Para esto, según lo establecido en distintos trabajos (Dutrénit, Santiago-Rodríguez y Vera-Cruz, 2006; Fuentes y Ampudia, 2009; García, 2017; Llisterri, Pietrobelli y Larsson 2011), se seleccionaron diferentes indicadores usados en marcos de referencia que parten de las condiciones propias del sistema nacional de innovación en México y se adaptaron a las características de los subsistemas del SRI del país. También se consideró el ambiente institucional en el cual se desempeña el SRI como parte fundamental de la acción pública que busca influir en la dinámica económica y social del estado.

Siguiendo estudios en la misma línea (Fuentes y Ampudia, 2009; López, Moreno, Marín y Maldonado, 2013; Ordóñez, 2017), los resultados fueron interpre- 
tados a través de representaciones gráficas longitudinales y transversales. En cuanto al periodo de estudio, se decidió revisar los datos disponibles a partir de 2016, debido al limitado acceso a información pública estatal previa en materia de ciencia y tecnología. Dicho análisis consideró las instituciones y las organizaciones, estatales y nacionales, de mayor importancia para cooperar y transmitir recursos dentro de Tamaulipas.

Los resultados obtenidos permitieron detectar las principales deficiencias del sistema mediante la clasificación de fallas que proponen Tödtling y Trippl (2005) de los SRI. Ésta se compone de tres aspectos: debilidad institucional, ausencia de vinculación y bloqueo.

\section{El sistema regional de innovación en Tamaulipas}

Tamaulipas cuenta con una posición estratégica porque está ubicado al norte del país y comparte frontera con Nuevo León, San Luis Potosí, Veracruz y Texas. De acuerdo con el Consejo Nacional de Población (CONAPO), la población del estado era de 3661217 habitantes en 2018, que equivale a 2.9\% de la población nacional. La entidad aportaba 3\% del PIB nacional, lo que la colocó en la decimosegunda posición nacional en 2017 (véase tabla 2). Se ubica en una posición parecida en PIB per cápita y se encuentra por encima de la media nacional (INEGI, 2019a).

Tabla 2. Principales indicadores socioeconómicos de Tamaulipas

\begin{tabular}{|l|r|c|c|}
\hline \multicolumn{1}{|c|}{ Indicador } & $\begin{array}{c}\text { Valor } \\
\text { estatal }\end{array}$ & $\begin{array}{c}\text { Media o total } \\
\text { nacional }\end{array}$ & Posición \\
\hline PIB (millones a pesos corrientes, 2017) & 490819 & 567609 & $12^{\circ}$ \\
\hline PIB per cápita (pesos, 2017) & 167803 & 167618 & $15^{\circ}$ \\
\hline Tasa de desempleo (2017) & $3.80 \%$ & $3.30 \%$ & $28^{\circ}$ \\
\hline Índice de desarrollo humano (2012) & 7.58 & 7.46 & $9^{\circ}$ \\
\hline Unidades económicas (2014) & 104334 & 4230745 & $12^{\circ}$ \\
\hline Años promedio de escolaridad (2015) & 9.5 & 9.2 & $14^{\circ}$ \\
\hline Porcentaje de población analfabeta (2015) & 3.00 & 5.50 & $9^{\circ}$ \\
\hline Pobreza (porcentaje de la población, 2016) & 32.20 & 43.60 & $13^{\circ}$ \\
\hline Porcentaje de las viviendas con computadora (2018) & 45 & 45 & $16^{\circ}$ \\
\hline Porcentaje de las viviendas con internet (2018) & 56 & 53 & $13^{\circ}$ \\
\hline Porcentaje de las viviendas con celular (2018) & 79 & 74 & $9^{\circ}$ \\
\hline
\end{tabular}

Fuente: elaboración propia con datos del Consejo Nacional de Evaluación de la Política de Desarrollo Social (CONEVAL) (2019), CONAPO (2019), Instituto Mexicano de la Propiedad Industrial (IMPI) (2019), INEGI (2019a), México Cómo Vamos (2019) y el Programa de las Naciones Unidas para el Desarrollo (PNUD) (2015). 
La entidad está dotada de varios polos de desarrollo a lo largo de su geografía, con especialización en las industrias petroquímica, automotriz, electrónica y agropecuaria. Tiene un enorme potencial para la producción de energía, ya que posee a escala nacional una de las reservas más grandes de petróleo y de gas natural. La combinación de estos factores económicos, sociales, demográficos, históricos y culturales permiten caracterizar Tamaulipas como una región con aptitudes y capacidades propias que la diferencian del resto del país.

Retraso institucional en Tamaulipas: la política tecnológica regional

El marco institucional está formado por las instituciones que elaboran y dictan las normas que regulan el quehacer científico y tecnológico. Como en la mayoría de los estados del país, en Tamaulipas la estructura pública asociada con las actividades científicas y tecnológicas se focaliza en el gobierno del estado y se ejecuta mediante un organismo público. En este caso, el Consejo Tamaulipeco de Ciencia y Tecnología (COTACYT) es el que tiene entre sus funciones promover el desarrollo de la investigación científica y tecnológica en el estado (COTACYT, 2020). La Secretaría de Educación en Tamaulipas (SET) tiene una relación estrecha con las actividades de investigación y desarrollo (I+D), al ser la encargada de varios centros de educación tecnológica. La Secretaría de Desarrollo Económico (SDET), en teoría, debe propiciar las condiciones óptimas para potenciar y hacer competitivos los sectores productivos. También la Comisión de Innovación, Ciencia y Tecnología dentro del Congreso debe contribuir a regular y promover la actividad científica y de innovación. En esta comisión se analizan y se dictaminan las propuestas legislativas y los asuntos relacionados con estas actividades (Comisión de Innovación, Ciencia y Tecnología de Tamaulipas, 2019).

Los principales ejercicios de política pública existentes en materia de innovación son la Ley de Fomento de la Investigación Científica y Tecnológica (Congreso del Estado de Tamaulipas, 2004) y el Plan Estatal de Desarrollo 2016-2022 (Gobierno del Estado de Tamaulipas, 2017). Estos documentos proporcionan el marco legal para la identificación de posibles actividades de innovación y de sectores de especialización, así como para la construcción de las políticas de ciencia y tecnología. Sin embargo, no se aprecian ejercicios de ese tipo en ninguna de las administraciones estatales.

\section{Caracterización de la política tecnológica regional}

La política tecnológica regional en Tamaulipas ha estado limitada por los escasos esfuerzos de las instituciones estatales mencionadas para impulsar una estrategia de desarrollo económico. El COTACYT fue creado para ser el organismo articulador de la política tecnológica y coordinar dichos esfuerzos entre los actores del sistema. Sin embargo, desde su creación todas las propuestas han estado acotadas a los periodos sexenales de cada administración de gobierno y a la promoción de determinados programas con bajo impacto en la economía estatal. Los recursos presupuestados para estas áreas apenas se incrementaron 
8.40\% entre 2016 y 2020, mientras que el presupuesto total aumentó en el mismo periodo más de $36 \%$.

Tabla 3. Contribución del presupuesto estatal para ciencia, tecnología e innovación

(millones de pesos)

\begin{tabular}{|c|c|c|c|}
\hline Año & $\begin{array}{c}\text { Presupuesto } \\
\text { estatal total }\end{array}$ & $\begin{array}{c}\text { Fondos para ciencia, } \\
\text { tecnología e innovación }\end{array}$ & $\begin{array}{c}\text { Participación } \\
\text { porcentaje }\end{array}$ \\
\hline 2016 & $\$ 43652$ & $\$ 8.45$ & 0.019 \\
\hline 2017 & $\$ 46329$ & $\$ 8.09$ & 0.017 \\
\hline 2018 & $\$ 49858$ & $\$ 8.21$ & 0.016 \\
\hline 2019 & $\$ 54933$ & $\$ 9.34$ & 0.017 \\
\hline 2020 & $\$ 59513$ & $\$ 9.16$ & 0.015 \\
\hline
\end{tabular}

Fuente: elaboración propia con datos del Gobierno del Estado de Tamaulipas (2020).

La Secretaría de Desarrollo Económico de Tamaulipas dispone de programas especializados para la generación de condiciones propicias para la actividad empresarial (véase tabla 4). Estas propuestas se crean con base en objetivos a corto plazo, como favorecer el empleo. Dichos criterios atentan contra el éxito a largo plazo de la política de ciencia y tecnología, porque con cada cambio de gobierno se necesita reconsiderar desde lo fundamental las estrategias establecidas, para modificar y alterar la priorización de las áreas y de los sectores en función de los intereses de cada grupo. La mayoría de los programas se dividen en distintos esquemas de financiamiento enfocados en todo tipo de empresa. No obstante, existen proyectos de capacitación que incorporan una asesoría completa, desde la constitución legal hasta el desarrollo de productos o servicios con los estándares que demanda el mercado (véase tabla 4).

Tabla 4. Ejecución de la política tecnológica en Tamaulipas

\begin{tabular}{|c|c|c|}
\hline & Acciones & Tipo de apoyo \\
\hline 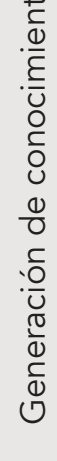 & $\begin{array}{l}\text { 1. Programas de licenciatura afines } \\
\text { 2. Pla ciencia y la tecnología. } \\
\text { Programa de registro de } \\
\text { propiedad intelectual. } \\
\text { 3. Programa Nacional de Posgrado } \\
\text { de Calidad (PNPC).* } \\
\text { 4. Becas nacionales del Consejo } \\
\text { Nacional de Ciencia y } \\
\text { Tecnología (CONACYT).* } \\
\text { 5. Capital científico (Sistema } \\
\text { Nacional de Investigadores).* }\end{array}$ & $\begin{array}{l}\text { 1. Acceso a estudios superiores en } \\
\text { ciencia e ingeniería. } \\
\text { 2. Asesoría en el registro de patentes } \\
\text { y modelos de utilidad. } \\
\text { 3. Programas de posgrados con altos } \\
\text { estándares de calidad. } \\
\text { 4. Financiamiento de PNPC. } \\
\text { 5. Plataforma para la formación y la } \\
\text { consolidación de investigadores. }\end{array}$ \\
\hline
\end{tabular}




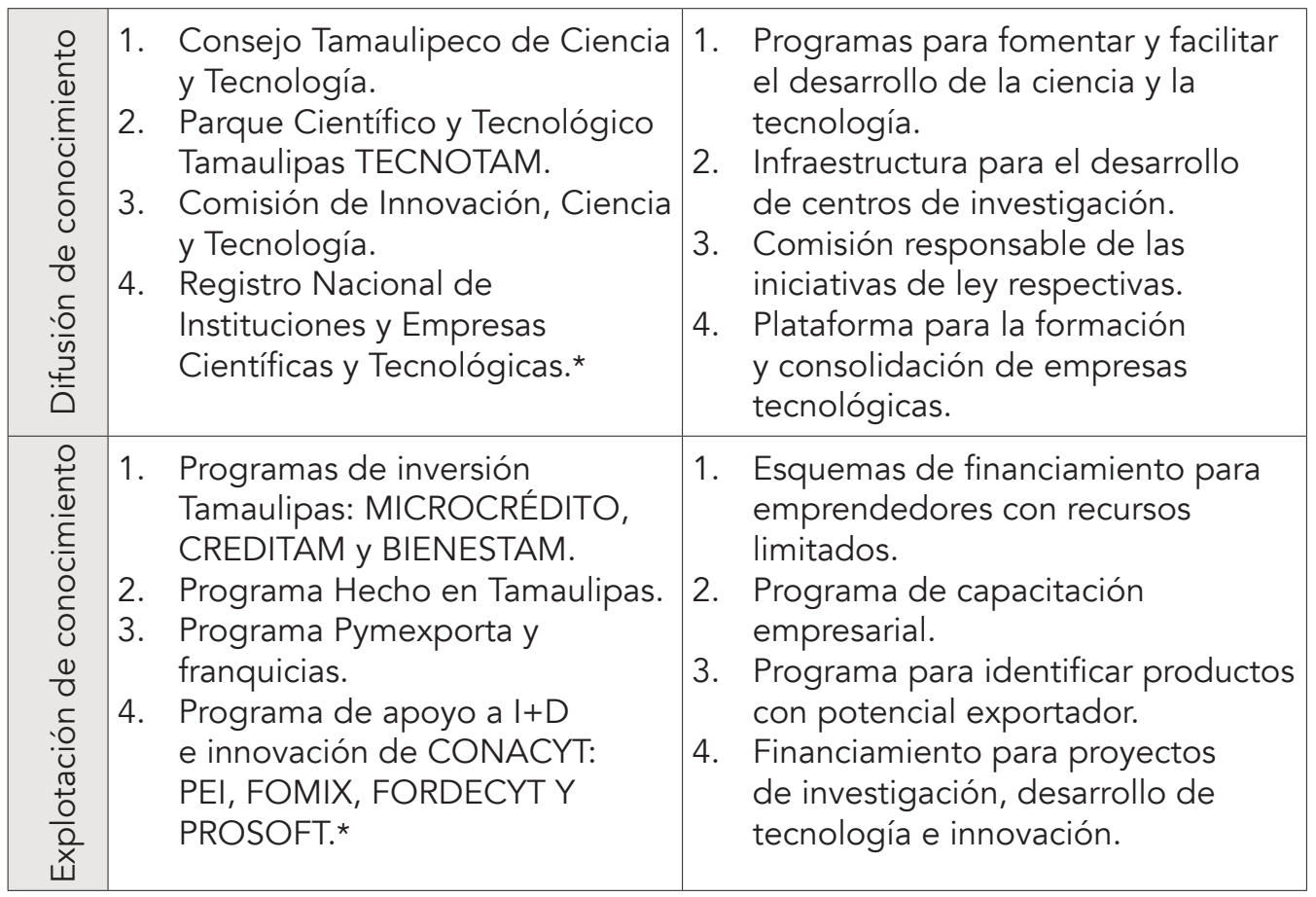

*Programas de origen nacional.

Fuente: elaboración propia.

Comunidad científica y tecnólogos: formación y producción de conocimientos

En el estado se encuentra un número relevante de instituciones destinadas a la investigación científica y al desarrollo tecnológico. Entre estas están los institutos tecnológicos y los institutos politécnicos, que tienen un papel esencial en la formación de profesionales calificados en las áreas de ingeniería. Una parte importante de los egresados de estas instituciones emigra a entidades colindantes o del centro del país, pues no encuentra en Tamaulipas opciones laborales acorde con su perfil profesional.

La Universidad Autónoma de Tamaulipas (UAT), institución pública de nivel superior, tiene 26 planteles distribuidos en ocho campus. La UAT es la organización que cuenta con el mayor número de alumnos de educación superior y con el mayor número de miembros del Sistema Nacional de Investigadores: 142 investigadores que pertenecen a las áreas de ciencias de la salud, ingeniería, medicina, agronomía, veterinaria y zootecnia, ciencias sociales y jurídicas (CONACYT, 2019a). En cuanto a los centros de investigación, sobresalen el Centro de Investigación de Estudios Avanzados (CINVESTAV) Tamaulipas, el Centro de Biotecnología Genómica (CBG), el Centro de Investigación en Ciencia Aplicada y Tecnología Avanzada (CICATA) Altamira y el Centro de Investigación Regional Noreste del Instituto Nacional de Investigaciones Forestales, Agrícolas y Pecuarias (INIFAP). 


\section{Capacidad de generación y conservación de talento}

En Tamaulipas, el número de alumnos matriculados en estudios de licenciatura aumentó $15 \%$ entre 2015 y 2018 . Durante el mismo periodo, los alumnos de programas de licenciatura afines a la CYT crecieron $6 \%$ y significaron $55 \%$ del total de matriculados para el ciclo 2018-2019 (véase figura 1). Además, el estado cuenta con un porcentaje superior de matriculados en programas afines a la CYT cuando se compara con la media nacional (1.9\% y $1.5 \%$ respectivamente) e inferior para el caso de los matriculados en posgrados $(0.2 \%$ y $0.3 \%$ ) (Asociación Nacional de Universidades e Instituciones de Educación Superior [ANUIES], 2020). En el caso de los posgrados, se ha experimentado una reducción de $14 \%$ de matriculaciones en los últimos años. En cuanto a los programas de CYT, la disminución entre 2015 y 2018 fue de $11 \%$ y supuso 9\% sobre el total de los alumnos de 2018 (ANUIES, 2020). La reducción en la matrícula de posgrado en general es reflejo de que una parte de la población formada opta por migrar y continuar sus estudios en otros estados o en el extranjero. Dicha reducción se debe quizás a tres factores: el primero es la inseguridad y violencia experimentada en años pasados. El segundo es el escaso número de empleos bien remunerado en comparación con los estados colindantes Nuevo León y San Luis Potosí (INEGI, 2019b). El tercero es una oferta de posgrado de mayor calidad y diversidad en otras entidades mexicanas.

Figura 1. Evolución de matriculaciones de licenciatura y posgrado
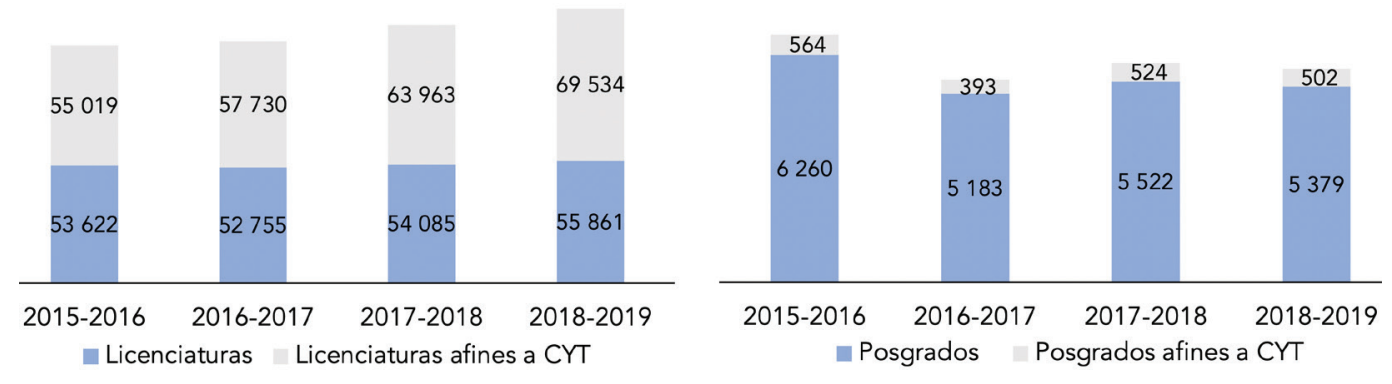

Fuente: elaboración propia con datos de la ANUIES (2020).

En 2018 las instituciones educativas tamaulipecas tenían 36 posgrados (1.5\% del total nacional) inscritos en el Programa Nacional de Posgrado de Calidad (PNPC) (véase figura 2). De los 36 programas, 88\% se clasifica "en desarrollo y de reciente creación”, entre los cuales sólo hay 12 doctorados. Por otra parte, $64 \%$ de los PNPC se relacionan con la CYT, en las áreas de ingeniería, ciencias agropecuarias y biotecnología (CONACYT, 2020a). 
Figura 2. Evolución del PNPC y becas nacionales del CONACYT

Evolución de PNCP

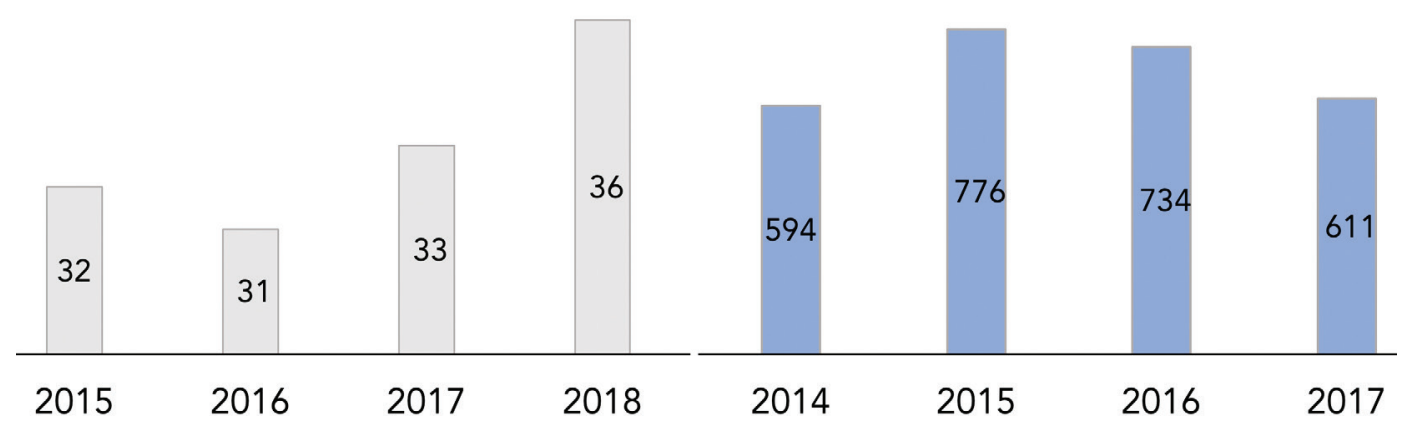

Fuente: elaboración propia con datos del CONACYT (2019b y 2020a).

En cuanto al número de becas nacionales que otorgó el CONACYT en 2017 (véase figura 2), Tamaulipas se colocó en la posición 19 a escala federal, con 611 becas vigentes (1.2\% del total nacional), lo que significa un desempeño inferior si se compara con su ratio de población (2.9\% del total nacional). Por áreas de conocimiento, las ingenierías significaron $25 \%$ de las becas otorgadas, seguidas por las ciencias sociales con 28\% (CONACYT, 2019b).

\section{Capacidades científicas y tecnológicas}

El desempeño de la entidad con respecto a la solicitud de patentes se sitúa por debajo de la media mexicana: 1.25 patentes por cada 100000 habitantes, en contraste con las 1.27 de la nación. Sin embargo, Tamaulipas se coloca en el decimoprimer lugar de los estados con el mayor número de solicitudes (IMPI, 2019). Entre 2015 y 2018 se experimentó un crecimiento de casi 100\%, cuando se pasó de 22 a 45 patentes por año. A pesar de esta mejoría, el uso de éstas en el sector productivo es aún muy limitado (véase figura 6).

En relación con el Sistema Nacional de Investigadores (SNI), el número de miembros aumentó de manera considerable al pasar de 177 a 261 integrantes entre 2014 y 2017. No obstante, aún se encuentra por debajo en cuanto a investigadores SNI por millón de habitantes, con tan sólo 76, mientras que la media nacional es 222. De los 261 integrantes del SNI en el estado, el nivel de éstos es menor que el nivel nacional, con $7 \%$ en el nivel II y $3 \%$ en el nivel III (frente a 16\% y $8 \%$ de México, respectivamente) (CONACYT, 2019a).

Respecto a la distribución por área de conocimiento (véase figura 3), Tamaulipas tiene la misma proporción que México $(69 \%)$ de investigadores en áreas afines a la CYT (I: física, matemáticas y ciencias de la tierra, II: biología y química; III: medicina y ciencias de la salud; VI: biotecnología y ciencias agropecuarias; VII: ingenierías). Destacan las áreas VI y VII, que representan $48.7 \%$ de los miembros del SNI estatales (CONACYT, 2019a). 
Figura 3. Nivel de los investigadores del Sistema Nacional de Investigadores y distribución por área de conocimiento. Porcentajes de 2017
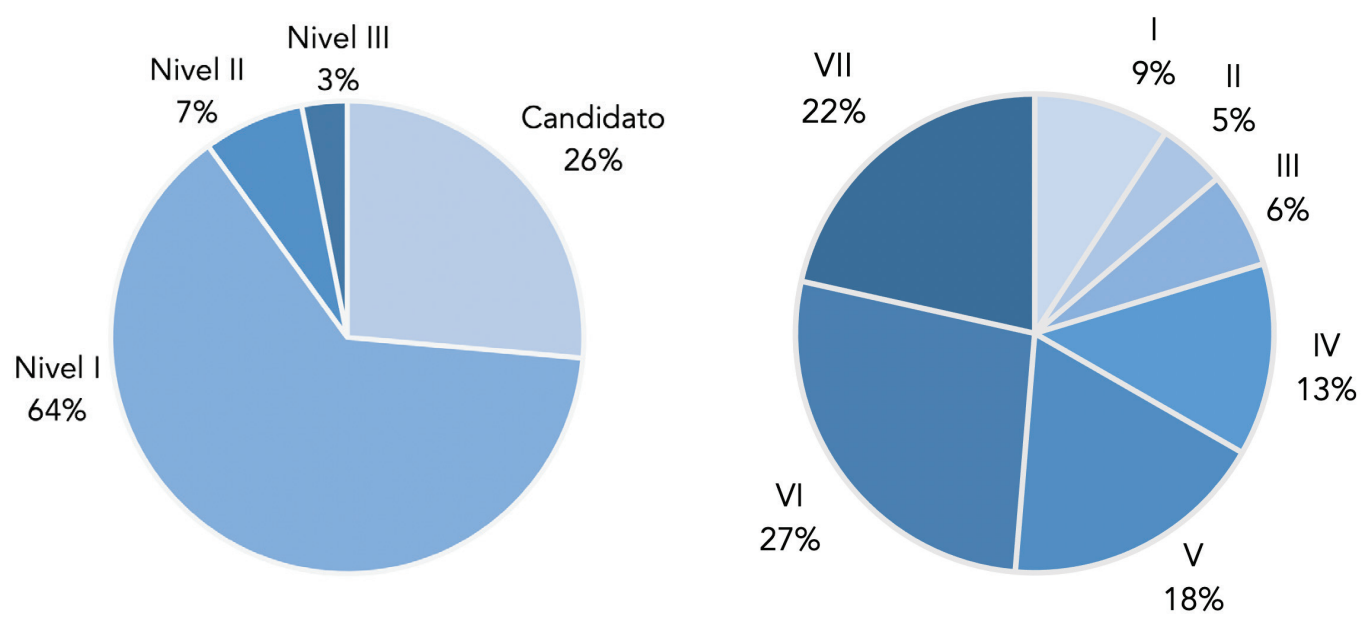

Fuente: elaboración propia con datos del CONACYT (2019a).

Sector productivo: especialización con bajo nivel tecnológico

La industria manufacturera tiene la mayor contribución al PIB estatal. Destacan la fabricación de maquinaria y equipo, así como la industria química y petroquímica (véase figura 4) (INEGI, 2019a). La actividad maquiladora mantiene un crecimiento moderado al concentrar $13 \%$ de los ingresos maquiladores de México. A pesar de la disminución de las unidades económicas desde la crisis de 2008, la fuerza laboral de la industria de manufactura ha aumentado en casi 22000 nuevos empleos (INEGI, 2020a).

En el contexto internacional, la entidad tenía una inversión extranjera directa de 1372 millones de dólares en 2018 que la ubicaba en la séptima posición nacional de mayor flujo de inversión, que en su mayoría provenía de Estados Unidos y se enfocaba en la industria manufacturera (61\%) (Secretaría de Economía, 2020). En lo referente a las exportaciones, Tamaulipas tiene una oferta basada en las industrias manufactureras de tecnología media y baja, dada la alta concentración de empresas maquiladoras. El principal subsector exportador es el de fabricación de equipo de computación, de comunicación y de medición, el cual significó 28\% de las exportaciones en 2019 (INEGI, 2019c). Cabe resaltar que estas actividades resultan de las operaciones de empresas extranjeras, que importan todo tipo de insumo y exportan su producción en los marcos de cadenas globales de valor. Por lo tanto, los beneficios para el estado son limitados, debido a la escasa integración que tienen con la economía local. 
Figura 4. Estructura del PIB de la industria manufacturera tamaulipeca por rama de actividad, porcentajes a precios constantes, 2017

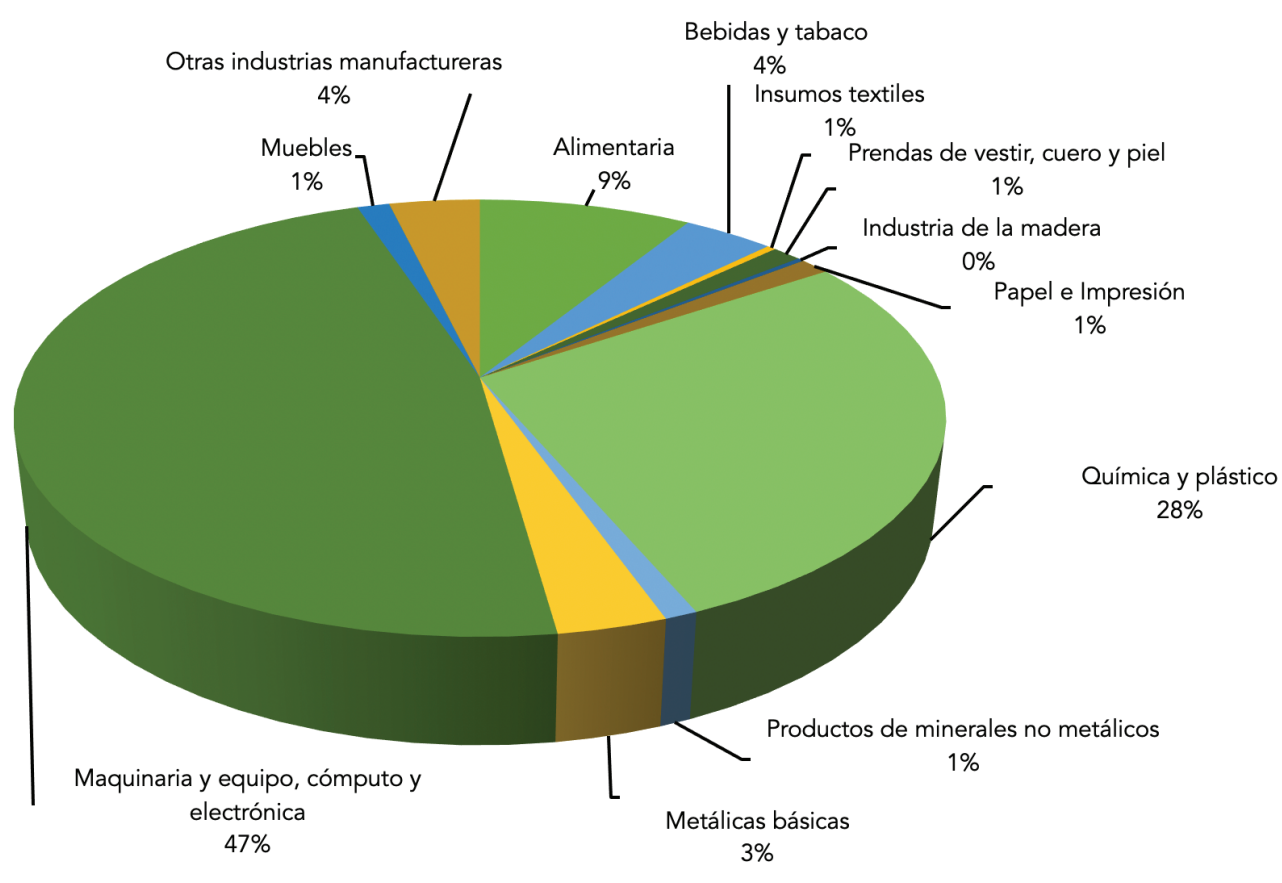

Fuente: elaboración propia con datos del INEGI (2019a).

El grado de especialización de las regiones que conforman Tamaulipas, las zonas fronteriza y sur son las que tienen una estructura económica similar a la estructura económica de la entidad (véase tabla 5), puesto que tanto la región fronteriza como la sur han basado su crecimiento en las industrias de alto crecimiento económico, como la petroquímica. En cambio, otras regiones, como el valle de San Fernando y el altiplano (más cercanas al coeficiente 1), tienen una estructura económica diferente (más cercanas al coeficiente 0 ), debido a que son zonas especializadas en la actividad agropecuaria.

Tabla 5. Coeficiente de especialización

\begin{tabular}{|c|l|l|l|}
\hline Región & 2004 & 2009 & 2014 \\
\hline Fronteriza & 0.07 & 0.11 & 0.16 \\
\hline Valle de San Fernando & 0.72 & 0.76 & 0.74 \\
\hline Centro & 0.4 & 0.56 & 0.5 \\
\hline Sur & 0.1 & 0.18 & 0.22 \\
\hline Mante & 0.28 & 0.47 & 0.43 \\
\hline Altiplano & 0.53 & 0.54 & 0.57 \\
\hline
\end{tabular}

Fuente: elaboración propia con datos del INEGI (2020b). 
Participación del sector productivo en el sistema regional de innovación

El Registro Nacional de Instituciones y Empresas Científicas y Tecnológicas (RENIECYT) es la plataforma que concentra las convocatorias de los distintos fondos de apoyos del CONACYT. Además, en ella se pueden conocer los indicadores con respecto al historial de apoyos y comportamiento de los beneficiarios. $\mathrm{El}$ RENIECYT en Tamaulipas ha crecido 25\% entre 2014 y 2017, y ha llegado a tener 181 entidades inscritas (1.5\% del total nacional). Sin embargo, este es un desempeño modesto, puesto que la media nacional es de 364 organizaciones (CONACYT, 2020b). En 2017, de las 181 inscripciones, 66\% pertenecían a empresas científicas y tecnológicas (véase figura 5), peso menor que la media federal (72\%). De la totalidad de empresas, las de mayor participación son las inscritas en el sector de los servicios profesionales, científicos y técnicos (25\%), seguidas por la industria de manufactura de maquinaria y equipo (15\%) y agricultura $(7 \%)$ (CONACYT, 2020b).

Figura 5. Distribución del RENIECYT por tipo de agente, 2017

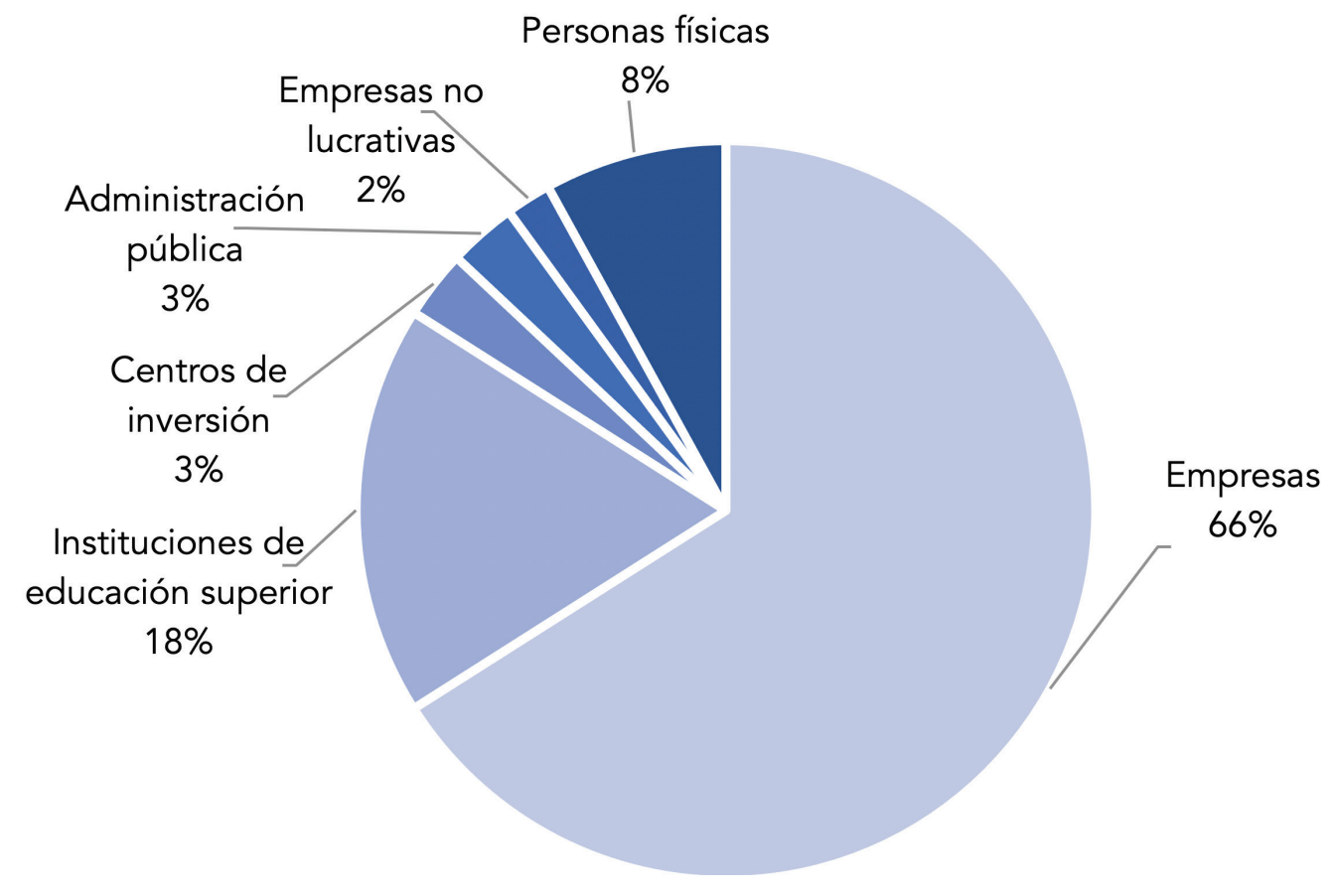

Fuente: elaboración propia con datos del CONACYT (2019b).

El nivel de madurez de la tecnología del tejido productivo tamaulipeco es insuficiente si se considera que de las empresas encuestadas por el INEGI sólo 251 realizaron con frecuencia alguna de las actividades de los nueve niveles de madurez tecnológica reconocidos, y 529 lo hicieron de forma esporádica. De nuevo estas cifras se encuentran por debajo de la media nacional (321 y 640, 
respectivamente) (INEGI, 2017). La misma institución ofrece información acerca de la actividad innovadora de las empresas del estado, en la cual se aprecia que las empresas desarrolladoras y vendedoras de tecnología se encuentran entre 2 y $12 \%$ del total (véase figura 6).

Figura 6. Empresas que realizan alguna de las actividades de madurez tecnológica, según la clasificación de madurez y constancia, 2017

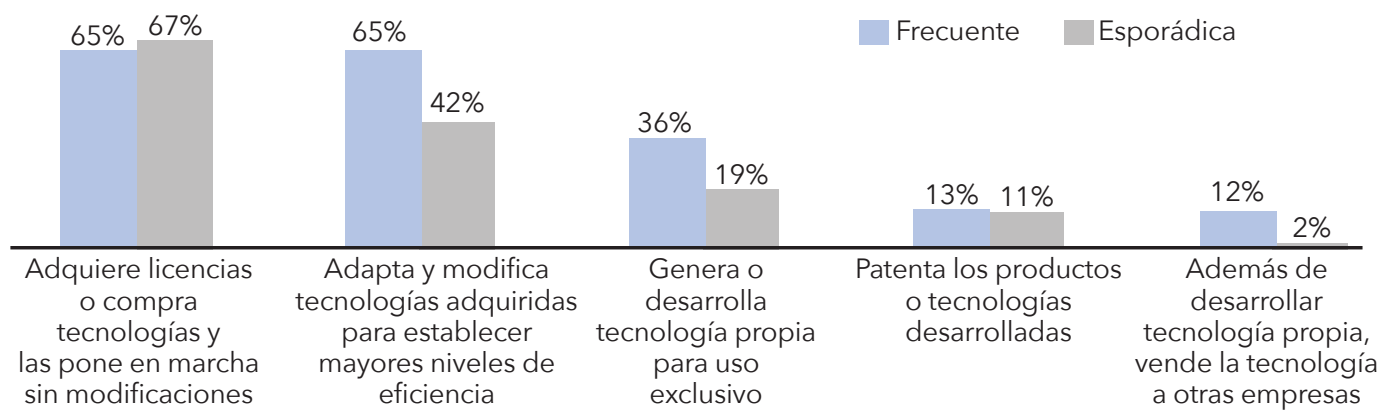

* La suma de los parciales no corresponde al total debido a que es una pregunta de opción múltiple. Fuente: elaboración propia con datos del INEGI (2017).

Figura 7. Distribución del PEl por sector entre 2010 y 2018

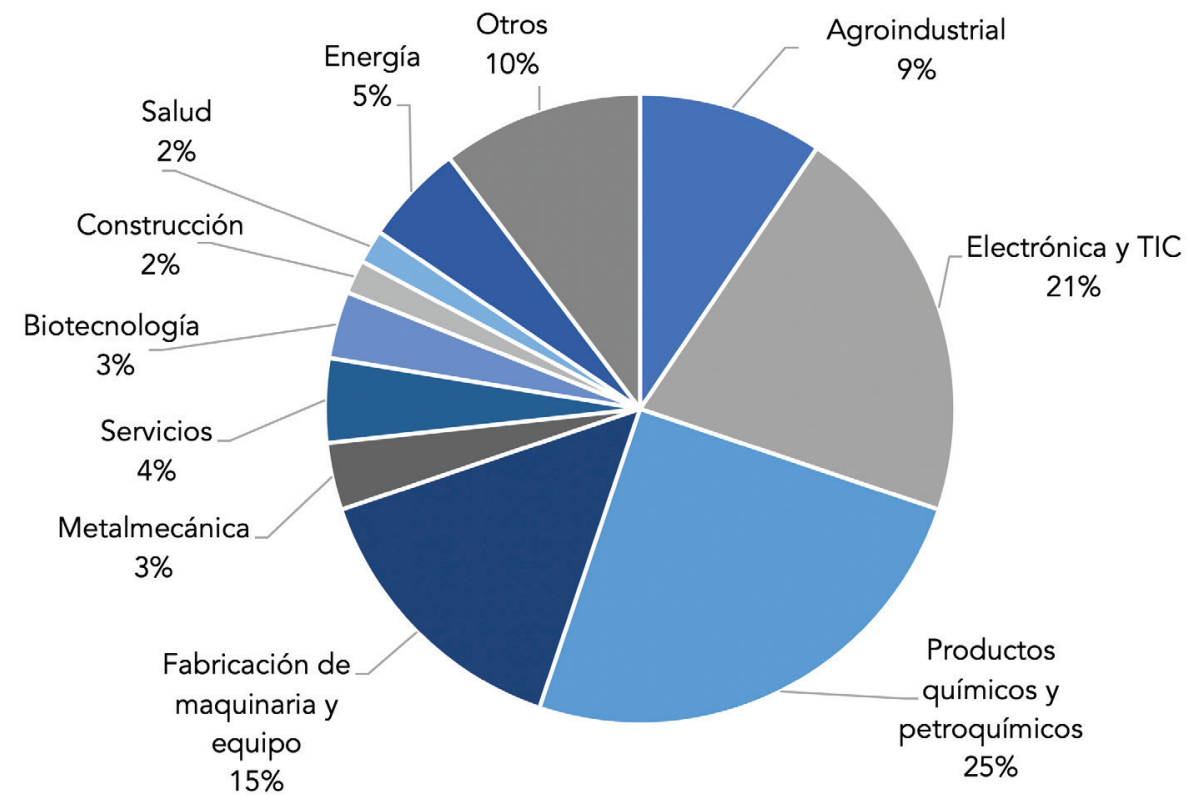

Fuente: elaboración propia con datos del CONACYT (2020c). 
A pesar del incremento en el registro de empresas de tipo científicas y tecnológicas, en los últimos años ha disminuido el número de dichas actividades. Este comportamiento se puede observar, de manera parcial, cuando se analizan los distintos programas de apoyo a la I+D, en especial el Programa de Estímulos a la Innovación (PEI), porque representa $81 \%$ del total de los financiamientos. Entre 2010 y 2018 se otorgaron 495 millones de pesos mediante el PEI (2.3\% del total nacional). En 2017 se apoyaron 11 empresas: siete proyectos en red orientados a la innovación (PROINNOVA), dos estímulos a la innovación (INNOVATEC), dos propuestas o proyectos de empresas micro, pequeñas y medianas (INNOVAPYME) (CONACYT, 2020c). En la figura 7 se presenta la distribución del monto con el que el PEl apoyó a los distintos sectores en esos años. De nuevo destacan el sector químico y petroquímico $(25 \%)$ y la industria electrónica $(21 \%)$.

Tamaulipas tiene capacidades productivas y tecnológicas acumuladas en la petroquímica secundaria, que es responsable de la transformación de productos básicos e intermedios. Este subsector se concentra en la zona sur de la entidad, aprovechando la infraestructura marítima de Tampico y Altamira. En 2014 había 77 empresas generadoras de 6651 empleos que manufacturaban cerca de $27 \%$ de los productos químicos y petroquímicos de México y $80 \%$ de las resinas termoplásticas. En 2018 la industria presentó un crecimiento moderado: registró 8407 empleos distribuidos en 80 unidades económicas, las cuales representaron $17 \%$ de la producción bruta estatal (INEGI, 2020b).

Esta situación podría guardar relación con la disminución de los apoyos del PEI a dicho sector en los últimos cinco años, que pasaron de 20.4 millones de pesos en 2014 a 2.7 millones en 2018 (CONACYT, 2020c). A pesar de esta reducción, resalta el apoyo de un millón de pesos al proyecto de síntesis de poliestireno expandido (EPS) biodegradable a partir de monómeros de fuentes renovables vía radicales libres en 2018. Este proyecto de Styropek México, Sociedad Anónima (S. A.) de Capital Variable (C. V.), se registró en asociación con el Instituto Tecnológico de Ciudad Madero y el Centro de Tecnología Avanzada (CIATEQ) (CONACYT, 2020c).

La industria eléctrico-electrónica y de las tecnologías de la información y la comunicación (TIC) dirige su producción a la manufactura de partes y componentes electrónicos. Algunos de los productos que se ensamblan son interruptores de circuitos electrónicos, transformadores e inductores. Además, fabrican herramientas eléctricas. Las TIC consideraban tanto equipos como desarrollo de software. Dichas actividades se dividían en 53 empresas durante 2014. Además, eran responsables de 46513 empleos directos, de los cuales $87 \%$ se concentraba en Matamoros y Reynosa. Sin embargo, el subsector cedería terreno en su posicionamiento nacional cuando de representar $11.6 \%$ de la producción bruta total bajó a 10\% (INEGI, 2020b).

Durante los cuatro años siguientes, Tamaulipas recibió el beneficio del PEI mediante un apoyo de 42.5 millones de pesos (CONACYT, 2020c), lo cual podría relacionarse con el $15 \%$ de incremento de las empresas, las cuales crearon 56963 empleos durante 2018 (INEGI, 2020b). En el mismo año, DAVAD, S. A. de 
C. V. recibió el apoyo de cerca de cuatro millones de pesos para un proyecto de un dispositivo electrónico de comunicación óptica bidireccional en medidores de energía eléctrica. Entre las instituciones vinculadas se encuentran: Universidad Popular Autónoma del Estado de Puebla, Instituciones Educativas del Desierto e Instituto Panamericano de Innovación y Tecnología (CONACYT, 2020c). Sobresale que no hay ninguna institución tamaulipeca entre los integrantes.

Fallas o principales problemas del SRI en Tamaulipas

El principal obstáculo del SRI es la falta de elementos de vinculación dentro de la estructura del sistema. A pesar de contar con diversos agentes especializados y reguladores de las actividades propias de cada uno de los subsistemas, hay nula coordinación entre los esfuerzos de éstos. En la mayor parte de los casos, dichos agentes realizan acciones individuales de bajo impacto fuera de su subsistema y con base en objetivos a corto plazo. Esto imposibilita la adecuada transferencia y explotación del conocimiento. La necesidad de instituciones de interfaz se acentúa cuando se analiza el retraso que sufre el estado debido a una política tecnológica que ha sustituido el desarrollo de las capacidades regionales de innovación por las acciones deliberadas del mercado y que no ha sabido transformar el conocimiento obtenido mediante el sistema de producción en ventajas competitivas para el subsistema de explotación.

Otro de los mayores impedimentos para la construcción de un SRI desarrollado es la ausencia de objetivos claros en las instituciones rectoras de la política de ciencia y tecnología. El COTACYT, por ejemplo, ha tenido un impacto inferior al esperado porque se limita a los programas de bajo uso del conocimiento que se genera en la entidad y que no brindan un real beneficio económico. Esta delgadez institucional se refleja en la disminución del presupuesto asignado para dichas actividades en los últimos años, que bajó de 0.019 a $0.015 \%$. De la misma manera, los programas gubernamentales se han dedicado al financiamiento empresarial y han dejado de lado acciones claves, como la incorporación de empresas locales a las cadenas productivas y su vinculación con posibles agentes que potencialicen sus capacidades.

La aglomeración de las empresas en los sectores eléctrico, electrónico y petroquímico en el norte y en el sur es una de las mayores fortalezas de Tamaulipas. Sin embargo, no son producto de las políticas de clústeres, sino un resultado espontáneo derivado de las necesidades de las empresas extranjeras para rentabilizar su capital y no el del país. Esta situación provoca una grave problemática, porque desincentiva las actividades de innovación en el sector productivo debido a la poca ventaja competitiva que implica la explotación de tecnología propia. Un ejemplo claro es que más de la mitad de las empresas en territorio tamaulipeco compran su tecnología debido a su perfil maquilador y no realizan adaptaciones de acuerdo con sus necesidades, mientras que $2 \%$ desarrolla, patenta y vende tecnología propia de forma esporádica (INEGI, 2017). A esto se le tiene que sumar la carente infraestructura y la falta de programas de apoyos en la actual política regional. 


\section{Conclusiones}

Siguiendo la literatura sobre los sistemas regionales de innovación, el estudio describió las principales características de los agentes en los subsistemas referentes al sector académico y productivo del estado. Además, se añadieron las instituciones gubernamentales como un elemento más, debido a que su quehacer es parte primordial para el funcionamiento del sistema.

Los resultados revelan que hay avances muy desiguales en el SRI de Tamaulipas hasta inicios de 2020. El subsistema de generación del conocimiento presenta activos que han estado en crecimiento, pero se ubican por debajo de la media nacional. Se aprecia que los centros de investigación y la Universidad Autónoma de Tamaulipas (la principal institución de educación superior del estado) han tenido adelantos en las acciones de investigación científica $y$, en algunos casos, de desarrollo tecnológico. Sin embargo, son limitados los recursos y el interés para producir programas de estudio e investigaciones que atiendan a los problemas de la entidad.

Localizado sobre todo en las fronteras norte y sur, el subsistema de explotación del conocimiento se especializa en las actividades de manufactura de uso poco intenso del conocimiento y de alta tecnología. Las industrias de mayor peso son la fabricación de maquinaria y equipo, eléctrico-electrónica, TIC y la industria química y petroquímica. Estos sectores son los que mayor financiamiento recibieron del PEI. Pero el interés de la planta productiva en la creación de vínculos con el sector académico no es suficiente, sobre todo en cuanto a la cantidad de actividades de investigación.

Las carencias más significativas del sistema se encuentran en el orden institucional, porque las políticas tecnológicas regionales no han tenido una implementación rigurosa ni un seguimiento adecuado ni una visión a largo plazo. Por eso no ha habido una estrategia de desarrollo económico estatal. Además, no existen instituciones de interfaz que conviertan el conocimiento obtenido en sistema de producción o en conocimiento aprovechable en el subsistema de explotación (véase figura 8). Por ejemplo, aunque hay un consejo estatal que se implica en la instrumentación de la política tecnológica, éste no tiene representantes de los subsistemas.

Sólo si se logra progresar en estas áreas, el funcionamiento del sistema y de la política de ciencia y tecnología podrá desarrollarse de forma autónoma del ciclo político, encarar los problemas internos de agencia y reducir las amenazas de los grupos de interés (Dutrénit et al., 2006). De esta manera se crearían las condiciones propicias para enfrentar los desafíos que plantea una sociedad cada vez más dependiente del conocimiento, como es el caso de la de Tamaulipas. 
Figura 8. Estructura del sistema regional de innovación en Tamaulipas

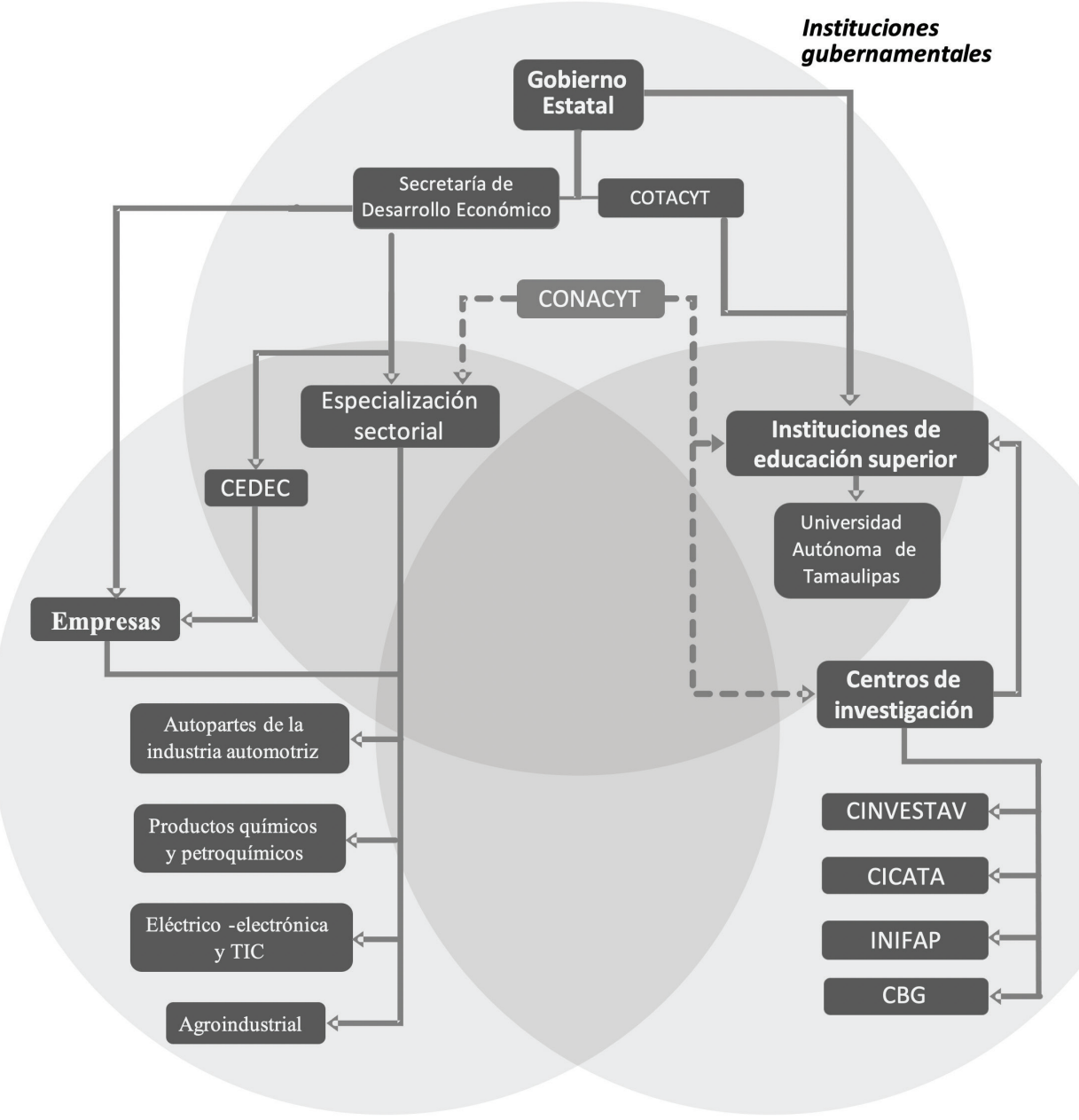

Subsistema de explotación del conocimiento

Subsistema de creación del conocimiento

CEDEC: Consejo Estatal para el Desarrollo Económico y la Competitividad.

Fuente: elaboración propia.

\section{Referencias}

Arrow, K. (1962). The economic implications of learning by doing. The Review of Economic Studies, 29(3), 155-173.

Asheim, B., e Isaksen, A. (1997). Location, agglomeration and innovation: towards regional innovation systems in Norway? European Planning Studies, 5(3), 299-330. doi: https://doi.org/10.1080/09654319708720402

Asociación Nacional de Universidades e Instituciones de Educación Superior

(ANUIES). (2020). Anuario Estadístico. Recuperado de http://www.anuies. $\mathrm{mx} /$ informacion-y-servicios/informacion-estadistica-de-educacion-superior 
Autio, E. (1998). Evaluation of RTD in regional systems of innovation. European Planning Studies, 6(2), 131-140. doi: https://doi. org/10.1080/09654319808720451

Carlsson, B. (2007). Innovation systems: a survey of the literature from a schumpeterian perspective. En H. Hanusch y A. Pyka (coord.), Elgar Companion to Neo-Schumpeterian Economics (pp. 857-871). Cheltenham y Northampton: Edward Elgar.

Chudnovsky, D. (1999). Políticas de ciencia y tecnología y el sistema nacional de innovación en la Argentina. Revista de la CEPAL, 153-171.

Cimoli, M., Ferraz, J., y Primi, A. (2007). Políticas de ciencia y tecnología en economías abiertas: la situación de América Latina y el Caribe. CEPAL-Serie Desarrollo Productivo, 165(1), 1-51.

Comisión Económica para América Latina y el Caribe (CEPAL). (2000). La política de desarrollo local en Italia: instrumentos y experiencias. Santiago: CEPAL. Recuperado de https://repositorio.cepal.org/bitstream/handle/11362/31419/S0005404_es. pdf?sequence=1\&isAllowed=y

Comisión de Innovación, Ciencia y Tecnología de Tamaulipas. (2019). Programa de trabajo. Recuperado de https://www. congresotamaulipas.gob.mx/Parlamentario/Archivos/Comisiones

Congreso del Estado de Tamaulipas. (2004). Ley 100 de 2004. Ley de Fomento a la Investigación Científica y Tecnológica en el Estado de Tamaulipas. Periódico Oficial del Estado de Tamaulipas, No. 16. Recuperado de http://po.tamaulipas.gob.mx/wp-content/uploads/2017/02/Ley_Investigacion_Cientifica.pdf

Consejo Nacional de Ciencia y Tecnología (CONACYT). (2019a). Sistema Nacional de Investigadores. Recuperado de https://www.conacyt.gob.mx/index. $\mathrm{php/el-conacyt/sistema-nacional-de-investigadores/archivo-historico}$

Consejo Nacional de Ciencia y Tecnología (CONACYT). (2019b). Actividad del CONACYT por Entidad Federativa 2017, Tamaulipas. Ciudad de México: CONACYT. Recuperado de https://www.siicyt.gob.mx/index.php/transparencia/ informes-conacyt/conacyt-en-las-entidades-federativas/conacyt-en-las-entidades-federativas-2017/4786-tamaulipas-2017/file

Consejo Nacional de Ciencia y Tecnología (CONACYT). (2020a). Padrón del Programa Nacional de Posgrados de Calidad. Recuperado de http://svrtmp. main.conacyt.mx/ConsultasPNPC/padron-pnpc.php

Consejo Nacional de Ciencia y Tecnología (CONACYT). (2020b). Sistema Integrado de Información sobre Investigación Científica, Desarrollo Tecnológico e Innovación. Recuperado de http://www.siicyt.gob.mx/index.php/k010-proyectos-de-infraestructura-social-de-ciencia-y-tecnologia/2-uncategorised/224-bases-de-datos-abiertas-otros

Consejo Nacional de Ciencia y Tecnología (CONACYT). (2020c). Dirección Adjunta de Desarrollo Tecnológico e Innovación. Recuperado de https: / /www. conacyt.gob.mx/Organigrama.html

Consejo Nacional de Evaluación de la Política de Desarrollo Social (CONEVAL). (2019). Medición de la pobreza. Recuperado de https://www.coneval.org. mx/Medicion/MP/Paginas/Pobreza-2018.aspx 
Consejo Nacional de Población (CONAPO). (2019). Proyecciones de población en México, 2010-2030. Recuperado de https://datos.gob.mx/busca/organization/conapo

Consejo Tamaulipeco de Ciencia y Tecnología (COTACYT). (2020). COTACYT. Recuperado de http://www.cotacyt.gob.mx/quienes-somos/

Cooke, P. (1992). Regional innovation systems: competitive regulation in the new Europe. Geoforum, 23(3), 365-382. doi: https: //doi.org/10.1016/00167185(92)90048-9

Cooke, P. (2001). Regional innovation systems, clusters, and the knowledge economy. Industrial and CorporateChange, 10(4), 945-974. doi: https://doi. org/10.1093/icc/10.4.945

Cooke, P., Roper, S., y Wylie, P. (2003). The golden thread of innovation and northern Ireland's evolving regional innovation system. Regional Studies (37), 365-379.

Cooke, P., Uranga, M., y Etxebarria, G. (1998). Regional systems of innovation: an evolutionary perspective. Environment and Planning, 30(9), 1563-1584. doi: https://doi.org/10.1068/a301563

Cooper, C. (1999). National Systems of Innovation: The Institutional Framework for Technological Learning in Developing Countries. Conferencia: Creating a new architecture for learning and development, por el Asian Development Bank. Tokyo.

Doloreux, D. (2002). What we should know about regional systems of innovation. Technology in Society, 24(3), 243-263.

Doloreux, D., y Porto, S. (2005). Regional innovation systems: current discourse and unresolved issues. Technology in Society, 27(2), 133-153.

Doloreux, D., y Porto, I. (2017). A review of (almost) 20 years of regional innovation systems research. European Planning Studies, 25(3), 371-387.

Dosi, G., Freeman, C., Nelson, R., Silverberg, G., y Soete, L. (1988). Technical Change and Economic Theory. Londres: Printer Publisher.

Dutrénit, G. (2009). Sistemas regionales de innovación: un espacio para el desarrollo de las pymes. Ciudad de México: Universidad Autónoma Metropolitana.

Dutrénit, G., Santiago-Rodríguez, F., y Vera-Cruz, A. O. (2006). Política de ciencia, tecnología e innovación, incentivos y comportamiento de los agentes: lecciones del caso mexicano. Economía: Teoría y Práctica, 24(1), 93-118.

Feria, M., Rodríguez, M., y Herrera, S. (2012). El sistema regional de innovación en Aguascalientes (México): entre el discurso y la realidad. Cuadernos de Administración, 25(45), 163-184.

Freeman, C. (1987). Technology Policy and Economic Performance: Lessons from Japan. Londres: Pinter Publisher LTD.

Fuentes, C., y Ampudia, L. (2009). Los sistemas regionales de innovación de Querétaro y Ciudad Juárez. En G. Dutrénit (coord.), Sistemas regionales de innovación: un espacio para el desarrollo de las PYMES (pp. 81-107). Ciudad de México: Universidad Autónoma Metropolitana.

García Fuentes, M. (2017). El enfoque de sistemas de innovación regionales: una crítica a su aplicación en México. Frontera Norte, 29(57), 177-186. 
Gobierno del Estado de Tamaulipas. (2017). Plan Estatal de Desarrollo 20162022. Gobierno del Estado de Tamaulipas. Recuperado de https://www.tamaulipas.gob.mx/planestatal/

Gobierno del Estado de Tamaulipas. (2020). Presupuesto de egresos del estado de Tamaulipas. Marco programático presupuestal. Recuperado de https:// transparencia.tamaulipas.gob.mx/transparencia-fiscal/marco-programati$\mathrm{col}$

Gu, S. (1999). Implications of national innovation systems for developing countries: managing change and complexity in economic development. Discussion Paper Series (03), 9-77.

Heijs. J. (2001). Sistemas nacionales y regionales de innovación y política tecnológica: una aproximación teórica. Documento de trabajo núm. 24, Universidad Complutense de Madrid. Recuperado de http://webs.ucm.es/BUCM/ cee/iaif/24/24.pdf

Instituto Mexicano de la Propiedad Industrial (IMPI). (2019). IMPI en cifras. Recuperado de https://www.gob.mx/impi/documentos/instituto-mexicano-de-la-propiedad-industrial-en-cifras-impi-en-cifras

Instituto Nacional de Estadística y Geografía (INEGI). (2017). Encuesta sobre Investigación y Desarrollo Tecnológico (ESIDET). Recuperado de https: / / www. inegi.org.mx/programas/esidet/2017/default.html\#Tabulados

Instituto Nacional de Estadística y Geografía (INEGI). (2019a). Sistema de cuentas nacionales de México. Recuperado de https://www.inegi.org.mx/temas/ $\mathrm{pib} /$

Instituto Nacional de Estadística y Geografía (INEGI). (2019b). Encuesta Nacional de Ocupación y Empleo (ENOE). Recuperado de https://www.inegi.org. $\mathrm{mx} / \mathrm{programas} / \mathrm{enoe} / 15 \mathrm{ymas} /$

Instituto Nacional de Estadística y Geografía (INEGI). (2019c). Exportaciones por entidad federativa. Exportaciones anuales por entidad federativa y subsector de actividad. Recuperado de https://www.inegi.org.mx/temas/ exportacionesef / default.html\#Tabulados

Instituto Nacional de Estadística y Geografía (INEGI). (2020a). Directorio Estadístico Nacional de Unidades Económicas (DENUE). Recuperado de https:// www.inegi.org.mx/app/mapa/denue/

Instituto Nacional de Estadística y Geografía (INEGI). (2020b). Censos Económicos. Recuperado de https://www.inegi.org.mx/app/saic/

Komninaki, D. (2015). Regional innovation systems in peripheral regions: insights from western Greece. Regional Studies, Regional Science, 2(1), 331339.

Llisterri, J., Pietrobelli, C., y Larsson, M. (2011). Los SRI en América Latina. Washington D. C.: Banco Interamericano de Desarrollo. Recuperado de https: / / publications.iadb.org/es/los-sistemas-regionales-de-innovacion-en-america-latina

López, V., Moreno, L., Marín, E., y Maldonado, S. (2013). Sistema regional de innovación: modelación de elementos desde el enfoque de sistemas, caso Baja California. Ciencia Administrativa, 30(1), 66-75. 
Lundvall, B. (1992). National systems of innovation: towards a theory of innovation and interactive Learning. Londres: Printer Publisher LTD.

Lundvall, B., Johnson, B., Andersen, E., y Dalum, B. (2002). National systems of production, innovation and competence building. Research Policy, 31(2), 213-231.

Lundvall, B., Vang, J., Joseph, K., y Chaminade, C. (2009). Innovation system research and developing countries. En Bengt-Åke Lundvall, K. J. Joseph, Cristina Chaminade y Jan Vang (eds.), Handbook of Innovation Systems and Countries. Building Domestic Capabilities in a Global Setting (pp. 1-30). Cheltenham: Edward Elgar.

Meyer-Krahmer, F. (1997). Technologiepolitik. En D. Ropohl y A. Schmid (cords.), Handbuch zur Arbeitslehre. Múnich: Oldenbourg.

Mas Verdú, F. (2014). Política de innovación y regiones: instrumentos y tendencias. En Diputación de Barcelona, Retos y futuro del desarrollo económico local (pp. 99-110). Barcelona: Serie Desarrollo Económico. Recuperado de https://asociacionredel.com/wp-content/libro/07.html

México Cómo Vamos. (2019). En cifras, ¿cómo vamos? Recuperado de mexicocomovamos.mx/encifrascomovamos.pdf

Morgan, K. (1997). The learning region: institutions, innovation and regional renewal. Regional Studies, 31(5), 491-503.

Nelson, R. (1992). National innovation systems: a retrospective on a study. Industrial and Corporate Change, 1(2), 347-374. doi: https://doi.org/10.1093/ icc /1.2.347

Niembro, A. (2019). Problemas y necesidades de los sistemas regionales de innovación en Argentina: hacia un enfoque territorial de las políticas de CTI. Redes, 25(48), 17-55.

Ordóñez, S. (2017). Sistemas de innovación y conocimiento: el caso de Jalisco, México. Problemas del Desarrollo, 48(191), 161-184. doi: https://doi. org/10.1016/j.rpd.2017.11.008

Organización para la Cooperación y el Desarrollo Económicos (OCDE). (2011). Regions and Innovation Policy. París: OECD Publishing.

Patel, P., y Pavitt, K. (2000). National system of innovation under strain: the internationalisation of corporate R\&D. En Barrell, Mason, O’Mahoney (coords.), Productivity, Innovation and Economic Performance (pp. 5-25). Cambridge: Cambridge University Press, .

Programa de las Naciones Unidas para el Desarrollo (PNUD). (2015). Índice de desarrollo humano para las entidades federativas, México. Ciudad de México: PNUD. Recuperado de https://www.mx.undp.org/content/mexico/es/ home/library/poverty/indice-de-desarrollo-humano-para-las-entidades-federativas--mexi.html

Quintero, L. (2010). Aportes teóricos para el estudio de un sistema de innovación. Innovar, 20(1), 57-76.

Rosales, S., y López, S. (2008). Base exportadora y sistema de innovación regional. El caso de Sinaloa. región y sociedad, 20(43), 163-187. doi: https://doi. org/10.22198/rys.2008.43.a500 
Secretaría de Economía. (2020). Inversión extranjera directa. Recuperado de https://www.gob.mx/se/acciones-y-programas/competitividad-y-normatividad-inversion-extranjera-directa?state=published

Solow, R. (1956). A contribution to the theory of economic growth. The Quarterly Journal of Economics, 70(1), 65-94.

Tödtling, F., y Trippl, M. (2005). Towards a differentiated regional innovation policy approach. Research Policy, 34(8), 1203-1219. doi: https://doi.org/10.1016/j.respol.2005.01.018

Tödtling, F., y Trippl, M. (2007). Developing biotechnology clusters in non-high technology regions -the case of Austria. Industry and Innovation, 14(1), 4767.

Tödtling, F., y Trippl, M. (2011). Regional innovation systems. En P. Cooke, B. Asheim, R. Boschma, R. Martin, D. Schwartz y F. Tödtling (coords.), Handbook of Regional Innovation and Growth (pp. 455-466). Cheltenham y Norththampton: Edward Elgar. 